\title{
A produção científica de docentes fonoaudiólogos de instituições públicas de ensino superior do Brasil
}

\author{
The scientific production of speech and language pathologists \\ professors linked to brazilian public higher education institutions
}

\begin{abstract}
Brunah de Castro Brasil ${ }^{1}$ - ${ }^{1}$ Universidade Federal do Rio Grande do Sul | Programa de Pós-Graduação em Educação em Ciências: Química da Vida e Saúde | Porto Alegre | RS | Brasil. Contato:

brasilbrunah@gmail.com. ORCID: http://orcid.org/0000-0002-9550-1988
\end{abstract}

Erissandra Gomes ${ }^{2}$ - ${ }^{2}$ Universidade Federal do Rio Grande do Sul | Programa de Pós-Graduação em Educação em Ciências: Química da Vida e Saúde | Porto Alegre | RS | Brasil. Contato: erifono@hotmail.com. ORCID: http://orcid.org/0000-0002-2379-7345

Maria do Rocio Fontoura Teixeira ${ }^{3}{ }^{3}$ Universidade Federal do Rio Grande do Sul | Programa de PósGraduação em Educação em Ciências: Química da Vida e Saúde | Porto Alegre | RS | Brasil. Contato: mrfontoura@gmail.com. ORCID: http://orcid.org/0000-0002-9888-7185

Resumo: A produção científica brasileira é resultado de pesquisas realizadas principalmente no sistema universitário do país. O objetivo deste estudo foi conhecer a produção científica dos docentes fonoaudiólogos vinculados aos cursos de Fonoaudiologia de instituições públicas de ensino superior do Brasil. Identificaram-se os 24 cursos vinculados a instituições de ensino superior públicas e os 383 docentes vinculados a elas. Utilizou-se o software Scriplattes para extração e análise dos dados relacionados à produção de artigos de periódicos, capítulos de livro e livros, a partir de dados disponíveis no currículo da Plataforma Lattes. Foram identificados, ainda, os periódicos, com as respectivas avaliações Qualis, fator de impacto e citescore, nos quais os docentes publicam suas pesquisas. Verificou-se que, no período de 2014 a 2018, foram publicados 2711 artigos em periódicos, 965 capítulos de livro e 130 livros, sendo alguns em colaboração entre os cursos. O periódico em que há maior número de publicações é a Revista CEFAC, a qual possui avaliação B1 no Qualis na área Educação Física (Fonoaudiologia), e não está indexada ao Journal Citation Reports ou a Scopus. Concluiu-se que (1) a produção científica dos docentes fonoaudiólogos é caracterizada principalmente pela publicação de artigos científicos e apresentou uma queda no período analisado; (2) há participação ativa da maioria das regiões do país na produção científica da área; e (3) os periódicos escolhidos pelos docentes para divulgação de seus estudos, em sua maioria, não possuem indexação a bases de dados internacionais e estão no estrato B do Qualis área Educação Física (Fonoaudiologia).

Palavras-chave: Fonoaudiologia. Ensino superior. Indicadores de produção científica.

Abstract: Brazilian scientific production is the result of research carried out mainly in the country's university system. The aim of this study was to know the scientific production of the speech and language pathologists professors linked to the courses of public higher education institutions in Brazil. The 24 courses were identified and the 383 professors linked to them were requested. Scriplattes software was used to extract and analyze data related to the production of journal articles, book chapters and books, from data available in the Lattes Platform curriculum. It was also identified in which journals the professors publish their research, as well as their evaluation. From 2014 to 2018, 2711 articles were published in journals, 965 book chapters and 130 books, some of them in collaboration between the courses. The journal with the largest number of publications is the Revista CEFAC, which has a Qualis B1 rating in the Physical Education area and is not indexed to the Journal Citation Reports or Scopus. It was concluded that the scientific production of speech, language and hearing sciences professors is mainly characterized by the publication of scientific articles, presented a decrease in the analyzed period, there is an active participation of most regions of the country in the scientific production of the area and the journals chosen for the dissemination of their articles are not indexed to international databases and are in the stratum B of the Qualis Physical Education area.

Keywords: Speech, language and hearing sciences. Higher education. Scientific publication indicators.

- Recebido em: 23 de outubro de 2019 - Aprovado em: 18 de junho de 2020

DOI: http://dx.doi.org/10.1590/S1414-40772020000300011

Este é um artigo publicado em acesso aberto sob uma licença Creative Commons https://creativecommons.org/licenses/by-nc/4.0/ 


\section{Introdução}

A produção científica representa os caminhos e os resultados alcançados pelas pesquisas ao longo dos anos, os quais são visíveis a partir da publicação e divulgação na comunidade científica. Os trabalhos são comumente publicados como artigos de periódicos, indexados a bases de dados, seguindo uma tendência observada em diversas áreas (OLIVEIRA; GRACIO, 2011). Os artigos em periódicos científicos são, então, a forma mais utilizada e reconhecida de divulgação da produção científica, seguidas dos livros (e capítulos), todos esses meios formais de divulgação que conseguem atingir um grande público (DANUELLO, 2014; FUNARO; NORONHA, 2006; MUELLER, 2000).

A avaliação da produção científica permite verificar, entre outros aspectos, a qualidade, competência e reconhecimento das pesquisas realizadas nos diferentes campos científicos. Ainda, é possível realizar um diagnóstico sobre a construção do conhecimento no campo e, assim, elaborar políticas públicas, focando nas potencialidades das instituições, pesquisadores e grupos de pesquisa. A divulgação dos trabalhos é fundamental não só para as pesquisas, mas também para a sociedade, uma vez que esta última investe nas instituições produtoras (OLIVEIRA; GRACIO, 2011; VOLPATO; FREITAS, 2003).

A produção científica brasileira representava 1\% da produção mundial em 2006 e atualmente chega a 2,5\%. Em relação à América Latina, os números são ainda maiores, chegando a 52,82\% no ano de 2018. O crescimento em relação à produção mundial, ainda que pequeno, mostra que o Brasil se preocupa com a produção de conhecimento gerada e entende como importante a sua divulgação (BEHLAU; GASPARINI, 2006; BRASIL, 2018).

A visibilidade da produção científica determina o quanto um estudo é capaz de influenciar a comunidade na qual está inserido e é possível medi-la considerando o impacto do estudo pelas citações aos periódicos indexados em bases de dados e a consequente avaliação de critérios quantitativos (ALMEIDA; GRÁCIO, 2019; DANUELLO; GUIMARÃES, 2005; DANUELLO; OLIVEIRA, 2012; FUNARO; NORONHA, 2006; PACKER; MENEGHINI, 2006). A cienciometria fornece resultados objetivos, permitindo considerar a construção do conhecimento científico no campo, bem como a dinâmica das pesquisas científicas no país. Para isso, faz uso de indicadores bibliométricos que expõem a tendência de comportamento do campo ao longo do tempo (DANUELLO, 2014; DANUELLO; GUIMARÃES, 2005; HAYASHI et al., 2005; PIZZANI et al., 2008; SAES, 2000).

O sistema universitário é o principal responsável pela produção de conhecimento, cuja construção ocorre junto a pesquisas desenvolvidas na graduação e na pós-graduação. 
Principalmente no sistema universitário público, alguns programas do Governo Federal incentivaram, ao longo da última década, o crescimento das pesquisas no ensino superior, direta ou indiretamente, como é o caso, por exemplo, do Ciências sem Fronteiras e do Programa de Reestruturação e Expansão das Universidades Federais (BRASIL, 2009, 2011; SOUZA, 2018).

As 15 universidades que mais produziram nos últimos seis anos são todas públicas e responsáveis por mais de 60\% da produção total do Brasil (CLARIVATE ANALYTICS, 2019). Souza (2018) relata que $89,24 \%$ da produção científica brasileira são oriundos do sistema universitário brasileiro, apresentando crescimento nos últimos anos, mesmo com as políticas apresentadas pelo Governo Federal.

No campo da Fonoaudiologia, estudos sobre produção científica de pesquisadores, grupos ou instituições ainda são incipientes, focados em cursos de graduação e programas de pós-graduação em Fonoaudiologia do país, os periódicos em que publicam e as temáticas de preferência (BRAGA; CHIARI; GOULART, 2014; DANUELLO; OLIVEIRA, 2012; HERCULANO; NORBERTO, 2009a, 2009b). Ainda, são comuns as revisões sobre produção bibliográfica nas diferentes especialidades do campo (CARVALHO; FRIEDMAN, 2013; COELHO; BRASOLOTTO; BEVILACQUA, 2012; CORREAA; WEBER; MAXIMINO, 2016; DAMASCENO; FRIEDMAN, 2011; HAYASHIDA et al., 2012; MARQUES; LEMOS, 2017; MUNHOZ et al., 2007; SILVA; FRIEDMAN, 2010; SOARES, 2016; TOMAZ-MORAIS et al., 2016). Por ser um campo relativamente novo, com reconhecimento da profissão em 1981, entende-se ser de grande importância conhecer a produção da área a fím de sustentar o crescimento e o reconhecimento das atividades realizadas ao longo dos anos, partindo do empirismo verificado no início das práticas até chegar à Fonoaudiologia baseada em evidências que se busca atualmente (AARÃO et al., 2011; BERBERIAN, 2001; BRASIL, 1981).

A partir disso, o objetivo deste estudo foi conhecer a produção científica dos docentes fonoaudiólogos vinculados a cursos de graduação em Fonoaudiologia de instituições de ensino superior (IESs) públicas do Brasil.

\section{Métodos}

Este estudo caracteriza-se como uma pesquisa documental e descritiva, e a análise dos dados foi realizada a partir de uma abordagem quantitativa (GERHARDT; SILVEIRA, 2009; GIL, 2008). Como o estudo utilizou dados secundários e não realizou pesquisa com seres humanos, não houve necessidade de aprovação em comitê de ética em pesquisa. 
Inicialmente foi realizada uma busca no Portal e-MEC (BRASIL, 2007) para identificação dos cursos Fonoaudiologia de IESs públicas no Brasil. Obteve-se, por meio dos Sistemas Eletrônicos de Informação ao Cidadão federal e estaduais, o nome dos docentes fonoaudiólogos vinculados a esses cursos. Somente no caso de uma universidade, o sistema de informação do estado redirecionou ao portal da transparência, e as informações foram encontradas. Todos os cursos $(n=24)$, distribuídos em 21 IESs, retornaram a solicitação e obteve-se um total de 432 docentes indicados. Organizou-se uma planilha contendo o nome de todos os docentes por curso e IES e, após, foi realizada a conferência acessando o currículo disponível na Plataforma Lattes, do Conselho Nacional de Desenvolvimento Científico e Tecnológico (CNPq), de cada um. Foram excluídos da amostra aqueles que eram professores, mas tinham outras graduações, como farmacêuticos, médicos, cientistas sociais, linguistas, entre outros ( $\mathrm{n}=21)$; possuíam vínculo como técnicos-administrativos com a instituição, como fonoaudiólogos ou outro ( $\mathrm{n}=11)$; possuíam vínculo como professores substitutos $(\mathrm{n}=12)$; estavam em afastamento em outra instituição $(n=1)$; não possuíam registro na Plataforma Lattes $(n=2)$ e; não possuíam qualquer registro de vínculo com as instituições $(n=2)$. Sendo assim, a amostra que fez parte do estudo, composta por docentes fonoaudiólogos das IESs públicas brasileiras, totalizou 383 docentes. Os cursos de Fonoaudiologia das IESs públicas no Brasil, seu tipo de administração, a localização, o ano de início do curso e o número de docentes vinculados estão dispostos no Quadro 1. 
Quadro 1 - Cursos de Fonoaudiologia de instituições públicas de ensino superior, tipo de administração, localização, ano de início do curso e número de docentes

\begin{tabular}{|c|c|c|c|c|c|}
\hline Curso/Instituição & Sigla & $\begin{array}{c}\text { Tipo de } \\
\text { Administração }\end{array}$ & Região & $\begin{array}{l}\text { Ano de } \\
\text { Início }\end{array}$ & $\begin{array}{l}\text { Número de } \\
\text { Docentes }\end{array}$ \\
\hline $\begin{array}{l}\text { Universidade Federal de Santa } \\
\text { Maria }\end{array}$ & UFSM & Federal & \multirow{5}{*}{ Sul } & 1972 & 15 \\
\hline $\begin{array}{c}\text { Fundação Universidade de } \\
\text { Ciências da Saúde de Porto } \\
\text { Alegre }\end{array}$ & UFCSPA & Federal & & 2007 & 14 \\
\hline $\begin{array}{c}\text { Universidade Federal do Rio } \\
\text { Grande do Sul }\end{array}$ & UFRGS & Federal & & 2008 & 16 \\
\hline $\begin{array}{c}\text { Universidade Federal de Santa } \\
\text { Catarina }\end{array}$ & UFSC & Federal & & 2009 & 19 \\
\hline $\begin{array}{l}\text { Universidade Estadual do } \\
\text { Centro Oeste }\end{array}$ & UNICENTRO & Estadual & & 2002 & 9 \\
\hline $\begin{array}{c}\text { Universidade Estadual de } \\
\text { Campinas }\end{array}$ & UNICAMP & Estadual & \multirow{10}{*}{ Sudeste } & 2002 & 11 \\
\hline $\begin{array}{c}\text { Universidade de São Paulo - } \\
\text { Campus Bauru }\end{array}$ & USP BAURU & Estadual & & 1980 & 25 \\
\hline $\begin{array}{l}\text { Universidade de São Paulo - } \\
\text { Campus Ribeirão Preto }\end{array}$ & $\begin{array}{c}\text { USP } \\
\text { RIBEIRÃO } \\
\text { PRETO } \\
\end{array}$ & Estadual & & 2003 & 9 \\
\hline $\begin{array}{c}\text { Universidade de São Paulo - } \\
\text { Campus São Paulo }\end{array}$ & $\begin{array}{l}\text { USP SÃO } \\
\text { PAULO }\end{array}$ & Estadual & & 1976 & 12 \\
\hline $\begin{array}{l}\text { Universidade Estadual Paulista } \\
\text { Júlio de Mesquita Filho }\end{array}$ & UNESP & Estadual & & 1988 & 11 \\
\hline $\begin{array}{l}\text { Universidade Federal } \\
\text { Fluminense }\end{array}$ & UFF & Federal & & 2010 & 12 \\
\hline $\begin{array}{l}\text { Universidade Federal do } \\
\text { Espírito Santo }\end{array}$ & UFES & Federal & & 2010 & 14 \\
\hline $\begin{array}{c}\text { Universidade Federal de Minas } \\
\text { Gerais }\end{array}$ & UFMG & Federal & & 2000 & 15 \\
\hline $\begin{array}{l}\text { Universidade Federal do Rio de } \\
\text { Janeiro }\end{array}$ & UFRJ & Federal & & 1994 & 22 \\
\hline $\begin{array}{l}\text { Universidade Federal de São } \\
\text { Paulo }\end{array}$ & UNIFESP & Federal & & 1968 & 16 \\
\hline Universidade de Brasília & UNB & Federal & $\begin{array}{l}\text { Centro- } \\
\text { Oeste }\end{array}$ & 2013 & 11 \\
\hline Universidade Federal da Bahia & UFBA & Federal & \multirow{8}{*}{ Nordeste } & 1999 & 20 \\
\hline $\begin{array}{c}\text { Universidade Federal de } \\
\text { Sergipe - Campus Aracajú }\end{array}$ & $\begin{array}{c}\text { UFS } \\
\text { ARACAJÚ }\end{array}$ & Federal & & 2007 & 12 \\
\hline $\begin{array}{l}\text { Universidade Federal de } \\
\text { Sergipe - Campus Lagarto }\end{array}$ & $\begin{array}{c}\text { UFS } \\
\text { LAGARTO }\end{array}$ & Federal & & 2011 & 14 \\
\hline $\begin{array}{c}\text { Universidade Estadual de } \\
\text { Ciências da Saúde de Alagoas }\end{array}$ & UNCISAL & Estadual & & 1997 & 26 \\
\hline $\begin{array}{c}\text { Universidade do Estado da } \\
\text { Bahia }\end{array}$ & UNEB & Estadual & & 1999 & 16 \\
\hline $\begin{array}{l}\text { Universidade Federal do Rio } \\
\text { Grande do Norte }\end{array}$ & UFRN & Federal & & 2009 & 17 \\
\hline $\begin{array}{c}\text { Universidade Federal da } \\
\text { Paraíba }\end{array}$ & UFPB & Federal & & 2009 & 23 \\
\hline $\begin{array}{c}\text { Universidade Federal de } \\
\text { Pernambuco }\end{array}$ & UFPE & Federal & & 1999 & 24 \\
\hline
\end{tabular}

Fonte: Elaborado pelas autoras (2019). 
Para acesso à produção dos docentes, optou-se pela análise dos currículos disponíveis na Plataforma Lattes, do CNPq, pois este se tornou padrão de registro das atividades acadêmicas em todo o país. Organizou-se uma matriz contendo o número identificador do currículo e o nome de cada docente, semelhante àquele do currículo, em um arquivo de texto, e utilizou-se o software ScriptLattes para extração dos dados a partir da matriz, no mês de julho de 2019. Este software fornece dados de produção bibliográfica, como artigos publicados em periódicos, capítulos de livro e livros, entre outros, em um período que pode ser determinado pelo pesquisador.

Os currículos analisados foram atualizados na Plataforma Lattes, em sua maioria no ano de 2019 ( $\mathrm{n}=311 ; 81,2 \%)$, sendo verificadas atualizações também nos anos de 2018 ( $\mathrm{n}=55$; 14,37\%), 2017 ( $n=9 ; 2,35 \%), 2016$ ( $n=5 ; 1,3 \%), 2014(n=2 ; 0,52 \%)$ e $2011(n=1 ; 0,26 \%)$. Sendo assim, considera-se que os dados analisados neste estudo refletem a produção científica atual dos docentes fonoaudiólogos vinculados às IESs públicas.

A produção científica foi analisada considerando todos os cursos e cada um individualmente, quanto ao número de artigos, capítulos de livro e livros publicados nos últimos cinco anos completos, ou seja, de 2014 a 2018. Optou-se por considerar a produção dos últimos cinco anos por se entender que esta reflete a produção real do docente na instituição à qual está vinculado, considerando que nos últimos anos diversos concursos públicos para carreira do magistério superior permitiram o ingresso de titulados da área. Foram ranqueadas as universidades e os pesquisadores considerando os aspectos elencados acima e excluídos os documentos duplicados. Aqueles publicados em colaboração entre os cursos que fizeram parte da amostra foram identificados quanto aos cursos, número e ano de publicações.

Alguns indicadores bibliométricos para produção de artigos científicos são bastante conhecidos, como, por exemplo, o fator de impacto e o CiteScore. O fator de impacto é um índice publicado no Journal Citation Reports (JCR), que tem como objetivo avaliar a qualidade das publicações, isto é, fornece informações sobre os periódicos indexados no Science Citation Index (SCI) e no Social Science Citation Index (SSCI) (GARFIELD, 1999, 2006; STREHL, 2005). O CiteScore é um índice que apresenta o mesmo objetivo, mas calculado a partir de uma fórmula diferente, sendo baseado nas citações aos periódicos indexados na base Scopus, da Elsevier (FERNANDEZ-LLIMOS, 2018). Ambos os índices fornecem dados quantitativos inferindo a importância acadêmica dos periódicos indexados, sendo que o fator de impacto considera as citações a publicações dos dois anos anteriores ao do cálculo e o CiteScore dos três anos anteriores. 
No Brasil, a análise, classificação e avaliação dos periódicos nos quais os pesquisadores das diferentes áreas publicam os resultados de suas pesquisas ocorre pelo Qualis Periódicos, criado pela Coordenação de Aperfeiçoamento de Pessoal do Ensino Superior (CAPES) em 1998, fazendo parte do sistema de avaliação dos programas de pós-graduação. O Qualis deve ser utilizado para avaliar um conjunto de pesquisadores, seja em uma instituição ou em um programa de pós-graduação (BARATA, 2016). Neste estudo, consideraram-se os dados do Qualis da área Educação Física, subárea Fonoaudiologia, da CAPES.

A partir de um extrato do software ScriptLattes, foi realizada uma análise de todos os artigos publicados pelo grupo, no período já referido, e dos periódicos nos quais estavam publicados. Após, foram planilhados os periódicos e identificadas suas avaliações pela CAPES (Qualis), JCR (fator de impacto), Scopus (CiteScore), bem como a língua de publicação e a origem. Foram excluídos os que haviam sido publicados em revistas que não possuíam International Standard Serial Number (ISSN).

Os dados coletados foram organizados e analisados no Microsoft Excel, no qual foram realizadas médias aritméticas e elaborados rankings a partir dos resultados encontrados e, também, calculadas frequências absolutas e relativas no que se refere à publicação de artigos em periódicos.

\section{Resultados e discussão}

Apresenta-se, no Gráfico 1, o total de artigos publicados em periódicos, capítulos de livro e livros por ano, no período de 2014 a 2018. O total de publicações no período analisado foi de 2711 artigos, 965 capítulos de livro e 130 livros. 


\section{Gráfico 1 - Número de publicações em artigos, capítulos de livro e livros, no Brasil, no período de 2014 a 2018}

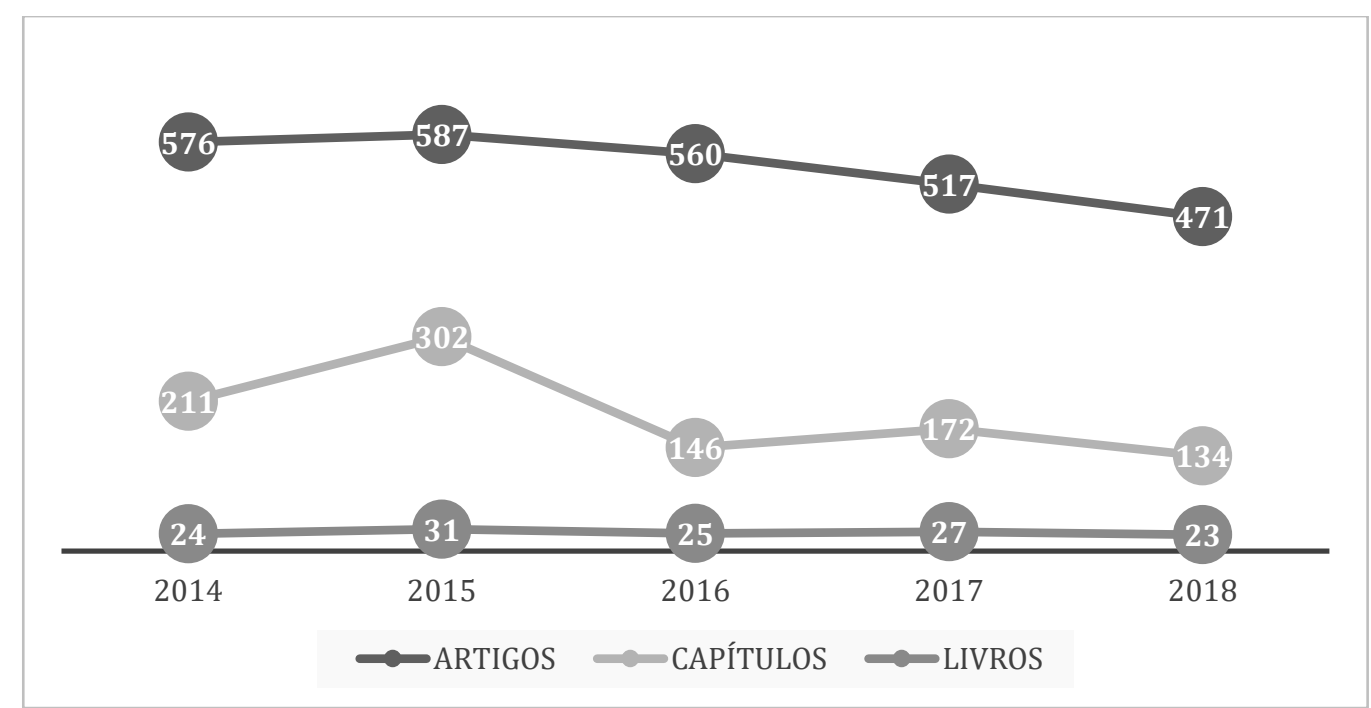

Fonte: Elaborado pelas autoras (2019).

Um número maior de artigos publicados, quando comparado à produção de capítulos de livro e livros corrobora dados de outros estudos, os quais analisaram a produção de programas de pós-graduação em Fonoaudiologia (BRAGA; CHIARI; GOULART, 2014; DANUELLO; OLIVEIRA, 2012). Há uma tendência de que as comunicações científicas ocorram comumente por meio de artigos, os quais proporcionam que os resultados encontrados em uma pesquisa cheguem mais rápido aos interlocutores, sendo, assim, o tipo de documento mais comumente indexado em bases de dados (DANUELLO; OLIVEIRA, 2012; OLIVEIRA; GRACIO, 2011).

Considerando os três tipos de documentos, o ano de 2015 foi o mais produtivo e, desde então, se observa uma queda na produção científica, principalmente no que se refere à produção de artigos, mas também - de forma mais discreta - para capítulos de livro, chegando, em 2018, à menor produção observada. Os artigos científicos são a forma mais comum de divulgação da produção e há um número maior de estudos sobre o assunto, por isso, a discussão trazida neste trabalho é principalmente sobre esse tipo de documento.

Ao contrário do que foi observado para os dados específicos da Fonoaudiologia, em 2018 o Brasil produziu mais de 50.000 artigos e obteve um crescimento de $30 \%$ no período de 2013 a 2018, quando a média mundial foi de 15\%, chegando à posição de líder em produção da América Latina (CLARIVATE ANALYTICS, 2019; GLÄNZEL; LETA; THIJS, 2006). É considerada como responsável por este crescimento a atuação de pesquisadores e instituições públicas, principalmente, com destaque para as agências de fomento e de regulação do ensino superior, como a CAPES e o Portal de Periódicos desta instituição, o qual proporciona o acesso a uma vasta bibliografia (ALMEIDA; GUIMARÃES, 2013; GLÄNZEL; LETA; THIJS, 2006; 
LETA; THIJS; GLÄNZEL; 2013). Além disso, o aumento das publicações em revistas indexadas a bases de dados internacionais também pode ter influenciado para que atualmente o país ocupe a $13^{\mathrm{a}}$ posição em produção científica entre todos os países do mundo (CLARIVATE ANALYTICS, 2019; CROSS; THOMSON; SINCLAIR, 2018).

Um dos aspectos que pode explicar a queda observada para a produção de artigos pelos docentes fonoaudiólogos é o fato de que o Governo Federal, financiador direto ou indireto das atividades das IESs que participaram deste estudo, reduziu os incentivos a partir de 2016. Essa redução fica caracterizada, principalmente, pela emenda constitucional 95/2016 (BRASIL, 2016), que congela os gastos em educação e saúde pelos próximos 20 anos para o pagamento da dívida do país. Essa mudança na política de governo em relação ao ensino superior faz com que a meta de se atingir $2 \%$ de investimentos em ciência e tecnologia até 2020, em uma tentativa de aproximação aos investimentos realizados por países desenvolvidos, não seja cumprida (CLARIVATE ANALYTICS, 2019; REGALADO, 2010). As atividades de pesquisa das IESs públicas sofreram mudanças nos últimos anos, essencialmente no que se refere a financiamento, como já exposto, mas também nas políticas de ciência e tecnologia, o que pode aparecer, em números, na queda de produção de artigos observada (SOUZA, 2018).

Os dados encontrados neste estudo, os quais evidenciam uma queda na produção de artigos dos docentes fonoaudiólogos a partir de 2016, estão de acordo com os indicadores de ciência, tecnologia e inovação de 2018 do Ministério da Ciência, Tecnologia, Inovações e Comunicações, os quais apontam uma redução da produção científica brasileira em relação à América Latina desde 2014 (BRASIL, 2018). A produção brasileira parece estar afetada por fatores como a força da economia do país, os financiamentos de pesquisa e a organização das IESs neste período de mudanças nas políticas públicas e de governo (CLARIVATE ANALYTICS, 2019).

Outro aspecto a ser levantado para justificar a queda diz respeito ao tempo de vinculação do docente ao curso, pois aquele que possui menos tempo de vínculo pode ter dificuldades de desenvolver atividades de pesquisa, seja por falta de financiamento seja por ligação a outras atividades de ensino ou extensão. Além disso, a amostra do estudo conta com docentes que atuam em cursos de graduação e muitos deles não estão inseridos em programas de pósgraduação, o que pode ser outro fator que explica a baixa de produção, uma vez que somente a atuação na graduação não exige aos docentes o mesmo de produção anual que os programas de pós-graduação estabelecem (HERCULANO; NORBERTO, 2009a). No último relatório da Web of Science (WoS) sobre a produção científica no Brasil, a área das Ciências da Saúde apresentou o menor crescimento de todas as áreas da CAPES no período de 2013 a 2018. As publicações 
desta área apresentaram impacto acima da média mundial e justificam o porquê de ser a área que mais produz, sendo responsável por 81.406 publicações nos últimos seis anos. Para as Ciências da Saúde, das 15 IESs mais produtivas e com maiores índices de impacto nas publicações, 11 e dez, respectivamente, fazem parte deste estudo, pois possuem curso de Fonoaudiologia, ou seja, a produção dos docentes fonoaudiólogos contribui para as estatísticas apresentadas pela CAPES, e os dados de queda de produção de artigos na amostra deste estudo corroboram os achados de baixo crescimento na área (CLARIVATE ANALYTICS, 2019).

Dos 2711 artigos publicados pelos docentes fonoaudiólogos, 236 foram produzidos em colaboração entre dois, três ou quatro dos cursos analisados neste estudo. Por isso, quando se observa a Tabela 1, o número total de artigos é maior (2962), uma vez que se apresenta o total de artigos produzidos por instituição. O mesmo é observado para capítulos de livro e livros, para os quais foram verificadas 88 e 15 colaborações, respectivamente. Na Tabela 1 também são apresentados os totais, por curso, de produção de capítulos de livro e livro.

Tabela 1 - Número de artigos, capítulos de livro e livros publicados por instituição, no Brasil

\begin{tabular}{|c|c|c|c|c|}
\hline Região & Instituição & Artigos & Capítulos de livro & Livros \\
\hline \multirow{5}{*}{ Sul } & UFSM & 328 & 38 & 3 \\
\hline & UFCSPA & 151 & 38 & 3 \\
\hline & UFRGS & 221 & 63 & 12 \\
\hline & UFSC & 122 & 35 & 6 \\
\hline & UNICENTRO & 60 & 19 & 1 \\
\hline \multirow{10}{*}{ Sudeste } & UNICAMP & 98 & 41 & 6 \\
\hline & USP BAURU & 268 & 96 & 14 \\
\hline & USP RIBEIRÃO PRETO & 83 & 20 & 2 \\
\hline & USP SÃO PAULO & 226 & 78 & 9 \\
\hline & UNESP & 185 & 67 & 24 \\
\hline & UFF & 33 & 23 & 3 \\
\hline & UFES & 24 & 17 & 2 \\
\hline & UFMG & 216 & 41 & 13 \\
\hline & UFRJ & 45 & 21 & 8 \\
\hline & UNIFESP & 78 & 71 & 4 \\
\hline Centro-Oeste & UNB & 57 & 16 & 0 \\
\hline \multirow{8}{*}{ Nordeste } & UFBA & 86 & 20 & 3 \\
\hline & UFS ARACAJÚ & 90 & 40 & 2 \\
\hline & UFS LAGARTO & 85 & 47 & 12 \\
\hline & UNCISAL & 79 & 26 & 3 \\
\hline & UNEB & 26 & 6 & 0 \\
\hline & UFRN & 86 & 39 & 4 \\
\hline & UFPB & 165 & 91 & 12 \\
\hline & UFPE & 150 & 109 & 17 \\
\hline Total & & 2962 & 1062 & 163 \\
\hline
\end{tabular}

Fonte: Elaborado pelas autoras (2019). 
Ao analisar as colaborações, verifica-se que, nos anos de 2014 e 2015, foram produzidos mais documentos em cooperação entre os cursos, cerca de $10 \%$ dos artigos e capítulos de livro e $22 \%$ dos livros. Os cursos que mais publicaram artigos e capítulos de livro em colaboração foram os da UFS - Campus Lagarto e Campus Aracaju, com 29 publicações de artigo (cerca de $32 \%$ do total de publicações no período) e 10 capítulos de livro (cerca de $23 \%$ do total de publicações). Essa colaboração pode ter sido mais facilitada, pois são dois cursos da mesma IES, embora estejam em campi diferentes, o que poderia favorecer a aproximação dos docentes. Porém, essa colaboração produtiva não foi observada para os três cursos da USP que fizeram parte deste estudo, por exemplo. É referido que as publicações apresentam mais impacto se forem realizadas em colaboração, nacional ou internacional (CLARIVATE ANALYTICS, 2019), sendo estas, então, sempre importantes para o desenvolvimento dos campos científicos.

Considerando as produções dos cursos, foram elaborados rankings para o total de publicações, bem como para a produção de artigos, capítulos de livro, livros, dispostos nas tabelas 2, 3, 4 e 5, respectivamente. Foram identificados alguns empates nos rankings, no que se refere ao número de publicações e, para apresentação, foi utilizada como critério de desempate a antiguidade do curso. Cabe ressaltar que, na Tabela 5, foram apresentados 11 cursos no ranking, pois ocorreu empate entre os cursos que ocupam a $10^{\mathrm{a}}$ e a $11^{\mathrm{a}}$ posições.

Tabela 2 - Ranking de produção científica no Brasil

\begin{tabular}{c|ccc}
\hline Posição & Instituição & $\begin{array}{c}\text { Número de } \\
\text { publicações }\end{array}$ & Região \\
\hline 1 & USP BAURU & 378 & Sudeste \\
2 & UFSM & 369 & Sul \\
3 & USP SÃO PAULO & 313 & Sudeste \\
4 & UFRGS & 296 & Sul \\
5 & UNESP & 276 & Sudeste \\
6 & UFPE & 276 & Nordeste \\
7 & UFMG & 270 & Sudeste \\
8 & UFPB & 268 & Nordeste \\
9 & UFCSPA & 192 & Sul \\
10 & UFSC & 163 & Sul \\
\hline
\end{tabular}

Fonte: Elaborado pelas autoras (2019). 
Tabela 3 - Ranking de produção de artigos no Brasil

\begin{tabular}{c|ccc}
\hline Posição & Instituição & Número de artigos & Região \\
\hline 1 & UFSM & 328 & Sul \\
2 & USP BAURU & 268 & Sudeste \\
3 & USP SÃO PAULO & 226 & Sudeste \\
4 & UFRGS & 221 & Sul \\
5 & UFMG & 216 & Sudeste \\
6 & UNESP & 185 & Sudeste \\
7 & UFPB & 165 & Nordeste \\
8 & UFCSPA & 151 & Sul \\
9 & UFPE & 150 & Nordeste \\
10 & UFSC & 122 & Sul \\
\hline
\end{tabular}

Fonte: Elaborado pelas autoras (2019).

Tabela 4 - Ranking de produção de capítulos de livro no Brasil

\begin{tabular}{c|ccc}
\hline Posição & Instituição & Número de capítulos de livro & Região \\
\hline 1 & UFPE & 109 & Nordeste \\
2 & USP BAURU & 96 & Sudeste \\
3 & UFPB & 91 & Nordeste \\
4 & USP SÃO PAULO & 78 & Sudeste \\
5 & UNIFESP & 71 & Sudeste \\
6 & UNESP & 67 & Sudeste \\
7 & UFRGS & 63 & Sul \\
8 & UFS LAGARTO & 47 & Nordeste \\
9 & UFMG & 41 & Sudeste \\
10 & UNICAMP & 41 & Sudeste \\
\hline
\end{tabular}

Fonte: Elaborado pelas autoras (2019).

Tabela 5 - Ranking de produção de livros no Brasil

\begin{tabular}{c|ccc}
\hline Posição & Instituição & Número de livros & Região \\
\hline 1 & UNESP & 24 & Sudeste \\
2 & UFPE & 17 & Nordeste \\
3 & USP BAURU & 14 & Sudeste \\
4 & UFMG & 13 & Sudeste \\
5 & UFRGS & 12 & Sul \\
6 & UFPB & 12 & Nordeste \\
7 & UFS LAGARTO & 12 & Nordeste \\
8 & USP SÃO PAULO & 9 & Sudeste \\
9 & UFRJ & 8 & Sudeste \\
10 & UNICAMP & 6 & Sudeste \\
11 & UFSC & 6 & Sul \\
\hline
\end{tabular}

Fonte: Elaborado pelas autoras (2019).

Considerando o total de produções, o curso que mais produz é o do Campus Bauru da USP. O curso de Fonoaudiologia da USP Bauru é um dos mais antigos do país e conta com um número de docentes acima da média nacional (de 15,96 docentes/curso), o que pode ser uma explicação para a alta produtividade. Não há dados na literatura sobre a produção geral da 
Fonoaudiologia, mas estudos de outras áreas referem que é na região sudeste que se concentra um maior número de cursos e, também, de produção científica (BRASIL; GOMES; TEIXEIRA, 2019; KOETZ; PÉRICO; GRAVE, 2017; SAES, 2000).

No que se refere à produção de artigos, a região sul aparece na liderança, representada pela UFSM, instituição com 328 artigos publicados no período analisado. O curso de Fonoaudiologia da UFSM, que foi um dos pioneiros no Brasil, possui um número de docentes próximo da média dos cursos de IESs públicas, mas o fato de contar com um programa de pósgraduação, ao qual quase a totalidade de seus professores é vinculada, pode justificar a alta produção de artigos científicos do grupo.

Contrariando os dados encontrados, Danuello e Oliveira (2012) identificaram o curso da USP Bauru como o mais produtivo quando analisaram as produções dos programas de pósgraduação em Fonoaudiologia do Brasil. Os achados deste estudo divergem, também, daqueles encontrados por Herculano e Norberto (2009a, 2009b), que referem que o curso do Campus São Paulo é o mais produtivo entre os cursos da USP. Cabe destacar que os três cursos citados (da UFSM, USP Bauru e USP São Paulo) aparecem nas três primeiras posições dos rankings dispostos nas tabelas 2 e 3 .

A região nordeste lidera o ranking de capítulos de livro publicados, representada pela UFPE, e a região sudeste, representada pela UNESP, lidera a produção de livros no país. Considerando que há cursos de Fonoaudiologia vinculados a IESs públicas em quatro regiões do país e três delas aparecem nos rankings, pode-se inferir que a produção está distribuída no país e que todas as regiões estão preocupadas com a divulgação dos resultados de suas pesquisas científicas. A única região que não aparece nos rankings elaborados é a Centro-Oeste, representada, na amostra, apenas por uma instituição, a UNB. Apesar de esta IES figurar entre as 15 mais produtivas do país (CLARIVATE ANALYTICS, 2019), o curso de Fonoaudiologia vinculado a ela foi o último a ser criado no país, em 2013 (BRASIL; GOMES; TEIXEIRA, 2019), e apresenta um número de docentes abaixo da média dos cursos de Fonoaudiologia de IESs públicas, o que pode justificar os baixos índices de produção observados. Vale lembrar que não há cursos de Fonoaudiologia de IESs públicas na região norte, por isso esta também não aparece nos rankings (BRASIL; GOMES; TEIXEIRA, 2019).

Torna-se importante analisar a publicação de artigos pela importância que apresenta na comunidade científica. Verificou-se que os artigos foram publicados em 525 diferentes periódicos, porém a produção se concentra em um número menor de revistas, visto que, em 62,28\% dos periódicos analisados, há apenas uma publicação dos docentes que participaram do estudo. Por isso, para fins de análise, organizou-se o Quadro 2, no qual são apresentados os dez 
periódicos que possuem o maior número de artigos publicados e, juntos, são responsáveis por $59,24 \%$ do total de produção do período de 2014 a 2018. Outros estudos também verificaram uma concentração da produção científica variando de 50 a 70\%, aproximadamente, em poucos periódicos (BRAGA; CHIARI; GOULART, 2014; DANUELLO; OLIVEIRA, 2012; HERCULANO; NORBERTO, 2009a).

Dos dez periódicos em que há maior número de publicações, apenas um é internacional, evidenciando uma tendência desses pesquisadores por publicações em periódicos brasileiros, corroborando dados de outros estudos (ALMEIDA; GUIMARÃES, 2013; BRAGA; CHIARI; GOULART, 2014; GLÄNZEL; LETA; THIJS, 2006; HERCULANO; NORBERTO, 2009a, 2009b; LETA, 2012). Essa tendência acarreta uma menor visibilidade de suas publicações e consequentes índices de citação baixos (GLÄNZEL; LETA; THIJS, 2006).

Quadro 2 - Periódicos com maior concentração de publicações e suas avaliações pelo Qualis, Fator de Impacto e CiteScore

\begin{tabular}{|c|c|c|c|c|c|c|c|}
\hline ISSN & Periódico & Origem & $\begin{array}{l}\text { Língua de } \\
\text { publicação }\end{array}$ & $\begin{array}{l}\text { Número } \\
\text { de } \\
\text { artigos }\end{array}$ & Qualis & $\begin{array}{c}\text { Fator } \\
\text { de } \\
\text { Impacto }\end{array}$ & CiteScore \\
\hline 1982-0216 & \multirow[b]{2}{*}{ Revista CEFAC } & \multirow[b]{2}{*}{ Nacional } & \multirow{2}{*}{$\begin{array}{c}\text { Inglês, } \\
\text { Português e } \\
\text { Espanhol } \\
\end{array}$} & \multirow[b]{2}{*}{479} & \multirow[b]{2}{*}{ B1 } & \multirow{2}{*}{ P } & \multirow[b]{2}{*}{$*$} \\
\hline 1516-1846 & & & & & & & \\
\hline $2317-1782$ & CODAS & Nacional & $\begin{array}{c}\text { Inglês, } \\
\text { Português e } \\
\text { Espanhol } \\
\end{array}$ & 341 & B1 & $*$ & 0,51 \\
\hline 2176-2724 & \multirow{2}{*}{$\begin{array}{l}\text { Revista Distúrbios da } \\
\text { Comunicação }\end{array}$} & \multirow[b]{2}{*}{ Nacional } & \multirow{2}{*}{\begin{tabular}{|c|} 
Inglês, \\
Português e \\
Espanhol \\
\end{tabular}} & \multirow[b]{2}{*}{271} & \multirow[b]{2}{*}{ B2 } & \multirow[b]{2}{*}{$*$} & \multirow[b]{2}{*}{$*$} \\
\hline $0102-762 X$ & & & & & & & \\
\hline $2317-6431$ & $\begin{array}{c}\text { Audiology - } \\
\text { Communication } \\
\text { Research }\end{array}$ & Nacional & $\begin{array}{l}\text { Inglês e } \\
\text { Português }\end{array}$ & 177 & B1 & $*$ & $*$ \\
\hline $1809-9777$ & \multirow{2}{*}{$\begin{array}{l}\text { International Archives } \\
\text { of Otorhinolaryngology }\end{array}$} & \multirow{2}{*}{ Nacional } & \multirow{2}{*}{ Inglês } & \multirow{2}{*}{118} & \multirow{2}{*}{ B1 } & \multirow{2}{*}{$*$} & \multirow{2}{*}{1,21} \\
\hline $1809-4864$ & & & & & & & \\
\hline $1808-8694$ & $\begin{array}{l}\text { Brazilian Journal of } \\
\text { Otorhinolaryngology }\end{array}$ & Nacional & Inglês & 91 & $\mathrm{~A} 2$ & 1,603 & 1,09 \\
\hline 0892-1997 & Journal of Voice & Internacional & Inglês & 64 & $\mathrm{~A} 2$ & 1,453 & 1,94 \\
\hline $1807-5932$ & Clinics & Nacional & Inglês & 26 & $\mathrm{~A} 2$ & 1,127 & 1,35 \\
\hline $1980-5764$ & $\begin{array}{c}\text { Dementia \& } \\
\text { Neuropsychologia }\end{array}$ & Nacional & Inglês & 20 & B2 & $*$ & 0,75 \\
\hline $1678-4464$ & \multirow{2}{*}{$\begin{array}{l}\text { Cadernos de Saúde } \\
\text { Pública }\end{array}$} & \multirow{2}{*}{ Nacional } & \multirow{2}{*}{$\begin{array}{c}\text { Inglês, } \\
\text { Português e } \\
\text { Espanhol } \\
\end{array}$} & \multirow{2}{*}{19} & \multirow{2}{*}{$\mathrm{A} 2$} & \multirow{2}{*}{1,17} & \multirow{2}{*}{1,02} \\
\hline $0102-311 X$ & & & & & & & \\
\hline
\end{tabular}

Fonte: Elaborado pelas autoras (2019).

Legenda: *Revistas não indexadas.

Cabe ressaltar, porém, que, dos nove periódicos nacionais, quatro exigem que as publicações sejam exclusivamente na língua inglesa, o que pode facilitar o acesso às pesquisas 
apresentadas. Leta (2012) verificou que as publicações na língua inglesa diminuíram depois que periódicos brasileiros que aceitam publicações na língua de origem foram indexados a WoS. A internacionalização dos periódicos brasileiros é muito importante para o desenvolvimento da produção científica no país e isso se torna possível não só com a indexação de periódicos a bases de dados internacionais, mas também com a publicação de estudos em inglês e o domínio dessa língua pelos pesquisadores (LETA, 2012).

A indexação de periódicos brasileiros em bases de dados internacionais exige diversos critérios, como rigor metodológico, tipo de avaliação, entre outros, mas há periódicos qualificados para tal (PACKER; MENEGHINI, 2017), uma vez que, nos últimos anos, o número de revistas indexadas a bases como WoS e Scopus aumentou (ALPERIN, 2013; SOUZA, 2018). Cabe observar que a principal base de dados brasileira, a Scientific Electronic Library On-Line (SciELO), atualmente é indexada tanto à WoS como à Scopus, duas das bases mais reconhecidas mundialmente. Essa indexação foi possível, entre outros aspectos, pelos investimentos realizados pelo governo em pessoal e infraestrutura para que as publicações brasileiras tivessem uma visibilidade internacional (LETA, 2012). Segundo Leta, Thijs e Glänzel (2013), as publicações brasileiras ainda possuem pouca visibilidade internacional, mas com um crescimento discreto observado nos últimos anos, provavelmente devido à indexação das revistas a bases de dados internacionais.

Não basta produzir em quantidade, por isso também são analisados indicadores de impacto das produções, ou seja, a qualidade dos trabalhos publicados e sua visibilidade na comunidade científica. O Brasil produz muito, inclusive mais que outros países da América Latina, mas seus índices de impacto de citação são mais baixos (CLARIVATE ANALYTICS, 2019).

No diz respeito à avaliação dos periódicos, todos estão classificados segundo o Qualis área Educação Física (Fonoaudiologia), enquanto apenas quatro dos dez periódicos possuem indexação ao JCR e sete, ao CiteScore, da base Scopus. Alguns estudos apontam a escolha por periódicos indexados ao ISI/JCR (ALMEIDA; GUIMARÃES, 2013; LETA, 2012) e outros referem que a tendência é por periódicos não indexados a bases internacionais (BRAGA; CHIARI; GOULART, 2014; HERCULANO; NORBERTO, 2009a; 2009b).

Verificou-se que seis dos dez periódicos estão concentrados no estrato B do Qualis área Educação Física (Fonoaudiologia) (B1 e B2), e o restante apresenta classificação A2. Nesta área da CAPES, há um maior número de periódicos classificados como A1, A2 e B1, o que equivale a boas classificações (BARATA, 2016). Braga, Chiari e Goulart (2014) também encontraram um predomínio de publicação em periódicos classificados como B1 e B2 para 
docentes de um programa de pós-graduação e Pellizzon, Chiari e Goulart (2014) indicam um maior número de publicações de bolsistas produtividade do CNPq em periódicos classificados como B2, B1 e A2.

Os periódicos brasileiros apresentam bom desempenho nos índices das bases de dados e mostram potencial para tornarem-se de alto impacto (PACKER; MENEGHINI, 2017). Observando o Quadro 2, verifica-se que a qualidade dos periódicos vai aumentando à medida que diminui o número de publicações, ou seja, há um menor número de publicações nos periódicos mais bem avaliados. No ranking elaborado, verifica-se que as revistas que ocupam as posições mais baixas do Quadro 2 são as mais bem avaliadas, com Qualis A2 e indexação ao JCR e à Scopus.

Os quatro periódicos em que os docentes analisados mais publicam são revistas específicas da área, que possuem grande reconhecimento na Fonoaudiologia, o que pode explicar a grande concentração de artigos encontrada neste estudo, evidenciando uma tendência de encaminhamentos de trabalhos a estas revistas (HERCULANO; NORBERTO, 2009a). Cabe ressaltar que esses periódicos também aparecem em rankings de outros estudos (BRAGA; CHIARI; GOULART, 2014; HERCULANO; NORBERTO, 2009a).

\section{Conclusões}

Foi possível concluir, analisando-se os últimos cinco anos, que a produção dos docentes fonoaudiólogos vinculados a IESs públicas está voltada a artigos científicos, mais do que a capítulos de livro e livros. Essa produção apresenta-se em queda, podendo ser reflexo da situação estabelecida, desde 2016, de diminuição dos incentivos e cerceamento de recursos ao ensino superior, no Brasil.

Observaram-se, para todos os tipos de documentos publicados, trabalhos em cooperação entre os cursos, seja na mesma IES ou não. Das quatro regiões em que há cursos de Fonoaudiologia, três aparecem nos rankings elaborados para visualização dos cursos que mais produzem os documentos analisados, ou seja, parece haver uma preocupação de todo o país para a produção e divulgação de conhecimento à comunidade científica.

As publicações de artigos científicos ocorrem preferencialmente em periódicos nacionais que aceitam submissões em língua portuguesa, classificados no estrato B do Qualis/CAPES na área Educação Física (Fonoaudiologia), e que não estão indexados a bases de dados internacionais. Cabe ressaltar que isso ocorre mesmo a literatura referindo um 
aumento no número de periódicos brasileiros indexados a essas bases. Ainda, foi possível verificar que há uma grande concentração de publicações em poucas revistas.

Com base no exposto acima, faz-se uma reflexão, a partir dos dados da literatura consultada, de que a produção da Fonoaudiologia acaba por não ter grande visibilidade internacionalmente, o que, se ocorresse, proporcionaria avanço no campo, principalmente no que se refere às estratégias e técnicas utilizadas na prática fonoaudiológica, a qual, por muitos anos, foi baseada no empirismo e vem buscando respaldo em evidências científicas.

As instituições públicas apresentam papel fundamental na produção científica brasileira. Por isso, pode-se considerar que os dados obtidos neste estudo são representativos da Fonoaudiologia brasileira como um todo. Valendo-se dos incentivos - seja financeiro seja de recursos humanos -, os docentes pesquisadores realizaram estudos e ofereceram maior reconhecimento ao campo nesse período.

A cienciometria permitiu um diagnóstico da produção científica no campo da Fonoaudiologia, no que se refere aos docentes de IESs públicas e, a partir disso, pode-se traçar o panorama de possibilidades futuras, considerando a situação política e econômica do país.

\section{Referências}

AARÃO, Poliane Cristina de Lima et al. Histórico da Fonoaudiologia: relato de alguns estados brasileiros. Revista Médica de Minas Gerais, Belo Horizonte, v. 21, n. 2, p. 238 $244,2011$.

ALMEIDA, Catia Candida de; GRÁCIO, Maria Claudia Cabrini. Produção científica brasileira sobre o indicador "Fator de Impacto": um estudo nas bases SciELO, Scopus e Web of Science. Encontros Bibli: revista eletrônica de Biblioteconomia e Ciência da Informação, Florianópolis, v. 24, n. 54, p. 62-77, 2019.

ALMEIDA, Elenara Chaves Edler de; GUIMARÃES, Jorge Almeida. Brazil's growing production of scientific articles: how are we doing with review articles and other qualitative indicators? Scientometrics, Amsterdam, v. 97, n. 2, p. 287-315, 2013.

ALPERIN, Juan Pablo. Brazil's exception to the world-class university movement. Quality in Higher Education, Abingdon, v. 19, n. 2, p. 158-172, 2013.

BARATA, Rita de Cassia Barradas. Dez coisas que você deveria saber sobre o Qualis. Revista Brasileira de Pós-Graduação, Brasília, v. 13, n. 30, p. 13-41, 2016.

BEHLAU, Mara; GASPARINI, Gisele. Education of speech-language pathologists and audiologists in Brazil. Folia Phoniatrica et Logopaedica, Basel, v. 58, p. 14-22, 2006.

BERBERIAN, Ana Paula. Linguagem e Fonoaudiologia: uma análise histórica. Revista Distúrbios da Comunicação, São Paulo, v. 12, n. 2, p. 265-278, 2001. 
BRAGA, Maria Elisa Rangel; CHIARI, Brasilia Maria; GOULART, Bárbara Niegia Garcia de. Produção bibliográfica em artigos, livros e capítulos de livros de um programa de pósgraduação em fonoaudiologia: análise de indicadores bibliométricos. Distúrbios da Comunicação, São Paulo, v. 26, n. 1, p. 118-130, 2014.

BRASIL. Decreto no 7.642, de 13 de dezembro de 2011. Institui o Programa Ciências sem Fronteiras. Brasília, DF, 2011. Disponível em:

http://www.cienciasemfronteiras.gov.br/documents/214072/5058435/Decreto7642-Csf.pdf. Acesso em: 10 mar. 2019.

BRASIL. Emenda Constitucional no 95, de 15 de dezembro de 2016. Altera o Ato das Disposições Constitucionais Transitórias, para instituir o Novo Regime Fiscal, e dá outras providências. Brasília, DF, 2016. Disponível em:

http://www.planalto.gov.br/ccivil_03/constituicao/emendas/emc/emc95.htm. Acesso em: 10 mar. 2019.

BRASIL. Lei $\mathbf{n}^{\mathbf{0}}$ 6.965, de 9 de dezembro de 1981. Dispõe sobre a regulamentação da profissão de Fonoaudiólogo, e determina outras providências. Brasília, DF, 1981. Disponível em: http://www2.camara.leg.br/legin/fed/lei/1980-1987/lei-6965-9-dezembro-1981-356567normaatualizada-pl.html. Acesso em: 20 ago. 2018.

BRASIL. Ministério da Ciência, Tecnologia, Inovações e Comunicações. Indicadores nacionais de ciência, tecnologia e inovação 2018. Brasília, DF: MCTIC, 2018. Disponível em:

https://www.mctic.gov.br/mctic/export/sites/institucional/indicadores/arquivos/Indicadores_C TI_2018.pdf. Acesso em: 13 set. 2019.

BRASIL. Portaria Normativa $\mathbf{n}^{\circ}$ 40, de 12 de dezembro de 2007. Institui o e-MEC, sistema eletrônico de fluxo de trabalho e gerenciamento de informações relativas aos processos de regulação da educação superior no sistema federal de educação. Brasília, DF, 2007.

Disponível em:

http://download.inep.gov.br/educacao_superior/censo_superior/legislacao/2007/portaria_40_1 2122007.pdf. Acesso em: 10 set. 2018.

BRASIL. Programa de Apoio a Planos de Reestruturação e Expansão das Universidades Federais Reuni 2008: relatório de primeiro ano. Brasília, DF: Ministério da Educação, 2009.

BRASIL, Brunah de Castro; GOMES, Erissandra; TEIXEIRA, Maria do Rocio Fontoura. O ensino de Fonoaudiologia no Brasil: retrato dos cursos de graduação. Revista Trabalho Educação e Saúde, Rio de Janeiro, v. 17, n. 3, p. 1-18, 2019.

CARVALHO, Audrey Vendramini de; FRIEDMAN, Silvia. Análise da produção científica internacional sobre gagueira. Revista CEFAC, São Paulo, v. 15, n. 5, p. 1236-1246, 2013.

CLARIVATE ANALYTICS. A pesquisa no Brasil: promovendo a excelência. [S. l.], 2019. Disponível em:

http://images.mail.discover.clarivate.com/Web/ClarivateAnalytics/\%7B1a55c3df-70a3-4ab18db4-d9741f748546\%7D_Web_of_Science_Group_Bibliometrics_Report_2019_PORT.pdf . Acesso em: 13 set. 2019. 
COELHO, Ana Cristina; BRASOLOTTO, Alcione Ghedini; BEVILACQUA, Maria Cecilia. Análise sistemática dos benefícios do uso do implante coclear na produção vocal. Jornal da Sociedade Brasileira de Fonoaudiologia, São Paulo, v. 24, n. 4, p. 395-402, 2012.

CORRÊA, Camila de Castro; WEBER, Silke Anna Theresa; MAXIMINO, Luciana Paula. Perfil da produção científica da apneia obstrutiva do sono na interface da Fonoaudiologia. Revista CEFAC, São Paulo, v. 18, n. 5, p. 1209-1221, 2016.

CROSS, Di; THOMSON, Simon; SINCLAIR, Alexandra. Research in Brazil: a report for CAPES by Clarivate Analytics. [S. l.], 2018. Disponível em: https://www.capes.gov.br/images/stories/download/diversos/17012018-CAPESInCitesReport-Final.pdf. Acesso em: 13 set. 2019.

DAMASCENO, Wladimir Alberti Pascoal de Lima; FRIEDMAN, Silvia. Análise da produção científica fonoaudiológica nacional sobre gagueira. Revista CEFAC, São Paulo, v. 13, n. 1, p. 41-47, 2011.

DANUELLO, Jane Coelho. Estudo da produção científica dos docentes de pós-graduação em Fonoaudiologia, no Brasil, para uma análise do domínio. 2014. Tese (Doutorado em Ciência da Informação) - Faculdade de Filosofia e Ciências, Universidade Estadual Paulista Julio de Mesquita Filho, Marília, 2014.

DANUELLO, Jane Coelho; GUIMARÃES, José Augusto Chaves. Produção científica docente em tratamento temático da informação nos cursos de Biblioteconomia do Mercosul: uma análise preliminar. Transinformação, Campinas, v. 17, n. 2, p. 153-168, 2005.

DANUELLO, Jane Coelho; OLIVEIRA, Ely Francina Tannuri de. Análise cientométrica: produção científica e redes colaborativas a partir das publicações dos docentes dos programas de pós-graduação em Fonoaudiologia no Brasil. Em Questão, Porto Alegre, v. 18, edição especial, p. 65-79, 2012.

FERNANDEZ-LLIMOS, Fernando. Differences and similarities between Journal Impact Factor and CiteScore. Pharmacy Practice, Granada, v. 16, n. 2, p. 1282, 2018.

FUNARO, Vânia Martins Bueno de Oliveira; NORONHA, Daisy Pires. Literatura cinzenta: canais de distribuição e incidência nas bases de dados. In: POBLACION, Dinah Aguiar; WITTER, Geraldina Porto; SILVA, José Fernando Modesto (org.). Comunicação e produção científica: contexto, indicadores e avaliação. São Paulo: Angelara, 2006. p. 215234.

GARFIELD, Eugene. Journal impact factor: a brief review. Canadian Medical Association Journal, Ottawa, v. 161, n. 8, p. 979-980, 1999.

GARFIELD, Eugene. The history and meaning of the Journal Impact Factor. Journal of the American Medical Association, Chicago, v. 295, n. 1, p. 90-93, 2006.

GERHARDT, Tatiana Engel; SILVEIRA, Denise Tofel. Métodos de pesquisa. Porto Alegre: Editora da UFRGS, 2009.

GIL, Antonio Carlos. Métodos e técnicas de pesquisa social. 6. ed. São Paulo: Atlas, 2008. 
GLÄNZEL, Wolfgang; LETA, Jacqueline; THIJS, Bart. Science in Brazil. Part 1: a macrolevel comparative study. Scientometrics, Amsterdam, v. 67, n. 1, p. 67-86, 2006.

HAYASHI, Maria Cristina Piumbato Innocentini et al. Competências informacionais para utilização da análise bibliométrica em educação e educação especial. Educação Temática Digital, Campinas, v. 7, n. 1, p. 11-27, 2005.

HAYASHIDA, Marina Zoega et al. Atenção Básica no SUS: publicações do Campus São Paulo da Unifesp, 1994-2009. Revista Brasileira de Educação Médica, Brasília, DF, v. 36, n. 3, p. 300-307, 2012.

HERCULANO, Rondinelli Donizetti; NORBERTO, Ana Maria Q. Uma análise percentual dos artigos de periódicos publicados pelos docentes de Fonoaudiologia da Universidade de São Paulo. Medicina, Ribeirão Preto, v. 42, n. 3, p. 372-378, 2009a.

HERCULANO, Rondinelli Donizetti; NORBERTO, Ana Maria Q. Comparison of scientific bibliographic productivity in undergraduate courses of speech-language and hearing science at Universidade of São Paulo using the Hirsh's index. Journal of Applied Sciences, New York, v. 9, n. 22, p. 4095-4098, 2009 b.

KOETZ, Lydia Chrismann Espíndola; PÉRICO, Eduardo; GRAVE, Magali Quevedo. Distribuição geográfica da formação em fisioterapia no Brasil: crescimento desordenado e desigualdade social. Trabalho, Educação e Saúde, Rio de Janeiro, v. 15, n. 3, p. 917-930, 2017.

LETA, Jacqueline. Brazilian growth in the mainstream science: The role of human resources and national journals. Journal of Scientometric Research, New Delhi, v. 1, n. 1, p. 44-52, 2012.

LETA, Jacqueline; THIJS, Bart; GLÄNZEL, Wolfgang. A macro-level study of science in Brazil: seven years later. Encontros Bibli: revista eletrônica de Biblioteconomia e Ciência da Informação, Florianópolis, v. 18, n. 36, p. 51-66, 2013.

MARQUES, Suzana Raquel Lopes; LEMOS, Stela Maris Aguiar. Instrumentos de avaliação do letramento em saúde: revisão de literatura. Audiology Communication Research, São Paulo, v. 22, p. 1-12, 2017.

MUELLER, Suzana Pinheiro Machado. O periódico científico. In: CAMPELLO, Bernadete Santos; CENDÓN, Beatriz Valadares; KREMER, Jeannette Margueriti (org.). Fontes de informação para pesquisadores e profissionais. Belo Horizonte: Editora UFMG, 2000. p. 73-95.

MUNHOZ, Cíntia Mara Affornalli et al. Análise da produção científica nacional fonoaudiológica acerca da linguagem escrita. Pró-Fono Revista de Atualização Científica, Barueri, v. 19, n. 3, p. 249-258, 2007.

OLIVEIRA, Ely Francina Tannuri de; GRACIO, Maria Cláudia Cabrini. Indicadores bibliométricos em Ciência da Informação: análise dos pesquisadores mais produtivos no tema estudos métricos na base de dados Scopus. Perspectivas em Ciência da Informação, Belo Horizonte, v. 16, n. 4, p. 16-28, 2011. 
PACKER, Abel; MENEGHINI, Rogerio. Visibilidade da produção científica. In:

POBLACION, Dinah Aguiar; WITTER, Geraldina Porto; SILVA, José Fernando Modesto. (org.). Comunicação e produção científica: contexto, indicadores e avaliação. São Paulo: Angelara, 2006. p. 235-259.

PACKER, Abel; MENEGHINI, Rogerio. The time has come for the quality journals of Brazil. SciELO in Perspective, São Paulo, 2017. Disponível em:

https://blog.scielo.org/en/2017/11/08/the-time-has-come-for-the-quality-journals-of-brazil/. Acesso em: 10 set. 2019.

PELLIZZON, Rosely de Fátima; CHIARI, Brasilia Maria; GOULART, Barbara Niegia Garcia de. Perfil dos pesquisadores com bolsa de produtividade em pesquisa do CNPq da área de Fonoaudiologia. Revista CEFAC, São Paulo, v. 16, n. 5, p. 1520-1532, 2014.

PIZZANI, Luciana et al. Um estudo bibliométrico da produção científica: a interface entre a educação especial e a fonoaudiologia nas bases de dados da Biblioteca Virtual em Saúde (BVS). Distúrbios da Comunicação, São Paulo, v. 20, n. 2, p. 205-218, 2008.

REGALADO, Antonio. Brazilian science: riding a gusher. Science, Washington, v. 330, n. 6009, p. 1306-1312, 2010.

SAES, Sueli Gonsalez. Estudo bibliométrico das publicações em economia da saúde, no Brasil, 1989-1998. 2000. Dissertação (Mestrado em Administração de Serviços de Saúde) Universidade de São Paulo, São Paulo, 2000.

SILVA, Marisa Cláudia Feital da; FRIEDMAN, Silvia. Análise da produção científica fonoaudiológica brasileira sobre paralisia cerebral. Revista da Sociedade Brasileira de Fonoaudiologia, São Paulo, v. 15, n. 4, p. 589-593, 2010.

SOARES, Marcelo Luiz Medeiros. Produção do conhecimento sobre educação popular e audiologia na atenção primária. Revista CEFAC, São Paulo, v. 18, n. 3, p. 789-800, 2016.

SOUZA, Claudia Daniele. Impacto de las políticas brasileñas de ciencia y tecnología en la actividad investigadora de las universidades federales: un estudio cienciométrico del período 2003-2015. 2018. Tese (Doctorado en Documentación: archivos y bibliotecas en el entorno digital) - Universidad Carlos III de Madrid, Madrid, 2018.

STREHL, Leticia. O fator de impacto do ISI e a avaliação da produção científica: aspectos conceituais e metodológicos. Ciência da Informação, Brasília, DF, v. 34, n. 1, p. 19-27, 2005.

TOMAZ-MORAIS, James et al. Análise integral da produção científica brasileira em motricidade orofacial: estado da arte e perspectivas futuras. Revista CEFAC, São Paulo, v. 18, n. 2, p. 520-532, 2016.

VOLPATO, Gilson Luiz; FREITAS, Eliane Gonçalves de. Desafios na publicação científica.

Pesquisa Odontológica Brasileira, São Paulo, v. 17, p. 49-56, 2003. Supl. 1. 